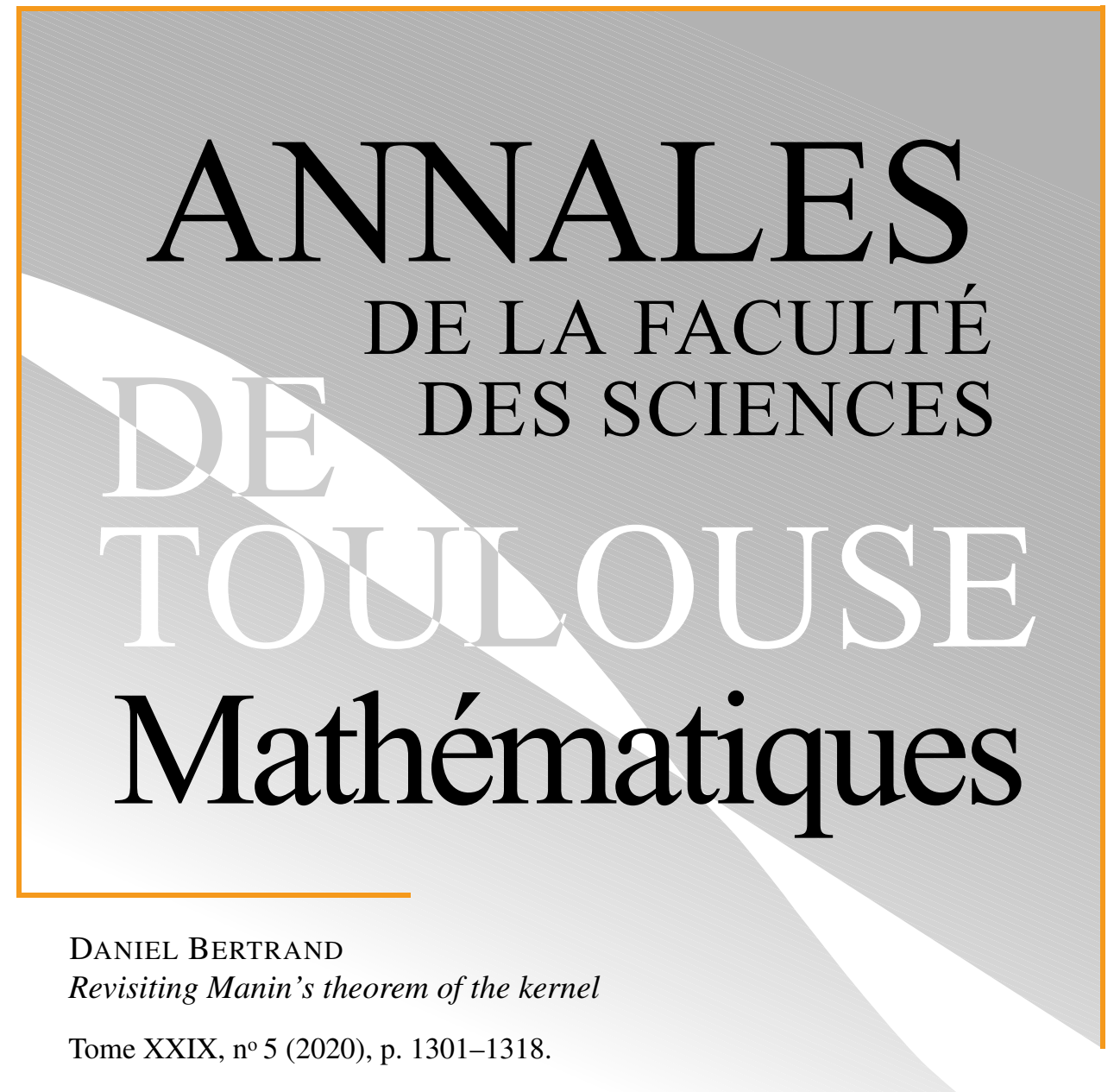

https://doi.org/10.5802/afst.1662

(C) Université Paul Sabatier, Toulouse, 2020.

L'accès aux articles de la revue «Annales de la faculté des sciences de Toulouse Mathématiques » (http://afst.centre-mersenne.org/) implique l'accord avec les conditions générales d'utilisation (http://afst.centre-mersenne.org/legal/). Les articles sont publiés sous la license CC-BY 4.0.

(c) $\underset{B Y}{(1)}$

Publication membre du centre

Mersenne pour l'édition scientifique ouverte MERSENNE http://www.centre-mersenne.org/ 


\title{
Revisiting Manin's theorem of the kernel
}

\author{
DANIEL BERTRAND ${ }^{(1)}$
}

À la mémoire de Hiroshi Umemura

\begin{abstract}
In the first part of the paper, we use Manin's map to establish a finiteness result linking rational sections of an elliptic scheme and solutions of Painlevé VI equations. The rest of the paper concerns abelian schemes over curves, and presents a survey of the various statements encompassed by Manin's theorem of the kernel.

RÉsumé. - Dans la première partie de ce texte, on établit au moyen de l'application de Manin un énoncé de finitude reliant les sections d'un schéma elliptique et les solutions des équations de Painlevé VI. Le reste de l'article concerne le théorème du noyau de Manin dans le cadre d'un schéma abélien sur une courbe, et passe en revue les divers énoncés connus sous cette appellation.
\end{abstract}

\section{A motivation from Painlevé VI equations}

\subsection{The Manin map in the elliptic case}

Consider the Gauss-Legendre elliptic scheme $(E): \eta^{2}=\xi(\xi-1)(\xi-t)$ over the complex curve $S=\mathbf{P}_{1} \backslash\{0,1, \infty\}$, and let the derivation $\partial=\mathrm{d} / \mathrm{d} t$ of $\mathcal{O}_{S}$ act on differential forms on $E / S$ by setting $\partial \xi=0, \partial(f \mathrm{~d} \xi)=\frac{\partial f}{\partial t} \cdot \mathrm{d} \xi$ for the coordinate system $(t, \xi)$. Then, $\omega=\frac{\mathrm{d} \xi}{\eta} \in H^{0}\left(E, \Omega_{E / S}^{1}\right) \subset H_{\mathrm{d} R}^{1}(E / S)$ satisfies

$$
L(\omega)=\frac{1}{2} d\left(\frac{\eta}{(\xi-t)^{2}}\right) \quad \text { where } \quad L:=t(1-t) \frac{\mathrm{d}^{2}}{\mathrm{~d} t^{2}}+(1-2 t) \frac{\mathrm{d}}{\mathrm{d} t}-\frac{1}{4} .
$$

Keywords: abelian varieties, Manin maps, Gauss-Manin connections, Mumford-Tate groups, Painlevé VI equations.

2020 Mathematics Subject Classification: 14K05, 32G20, 11G10, 12H05, 34M55.

(1) Sorbonne Université \& UMR $7586 \mathrm{du}$ CNRS, Institut de Mathématiques de Jussieu-PRG, Case 247, 75252 Paris Cédex 05, France - daniel.bertrand@imj-prg.fr 
So, the class of $L(\omega)$ in $H_{\mathrm{d} R}^{1}(E / S)$ vanishes, and the periods $\int_{\gamma} \omega, \gamma \in$ $H_{1, B}(E / S)$, generate over $\mathbb{C}$ the space of solutions of $L(\cdot)=0$ (note that $\left.\partial\left(\int_{\gamma} \omega\right)=\int_{\gamma} \partial \omega\right)$.

Let now $y(t):=(\xi(t), \eta(t)) \in E(S)$ be a section of $E / S$, and consider with Manin [18] the (multivalued) elliptic logarithm of $y$

$$
u(t)=\int_{0}^{y(t)} \omega=\ell n_{E}(y(t)) \in \operatorname{Lie} E\left(\widetilde{S}^{\text {an }}\right) .
$$

Since the indeterminacies of $u(t)$ are the periods of $\omega$, which are killed by $L$, and since $u(t)$ has moderate growth near its singularities, $L(u(t))$ is a well-defined element of the function field $K=\mathbb{C}(S)$, and in fact lies in $\mathcal{O}_{S}$. We therefore obtain a Manin map:

$$
\boldsymbol{\mu}_{E}: E(S) \longrightarrow \mathcal{O}_{S}: y \longmapsto \boldsymbol{\mu}_{E}(y):=L(u),
$$

which, taking into account the exact form above and fudge factors coming from derivation of the boundary ( $\partial$ no longer commutes with $\int_{0}^{y}$ : concretely, $\left.\partial\left(\int_{\infty}^{\xi(t)} f \mathrm{~d} \xi\right)-\int_{\infty}^{\xi(t)} \partial(f \mathrm{~d} \xi)=\xi^{\prime}(t) f(\xi(t))\right)$, is given by

$$
y=(\xi, \eta) \longmapsto \boldsymbol{\mu}_{E}(y)=\frac{1}{2} \frac{\eta}{(\xi-t)^{2}}+\left(t(1-t) \frac{\xi^{\prime}}{\eta}\right)^{\prime}+t(1-t) \frac{\xi^{\prime}}{\eta} \cdot \frac{\eta^{\prime}}{\eta},
$$

Since $\omega$ is an invariant form, $\boldsymbol{\mu}_{E}$ is a group homomorphism. Since the target space is uniquely divisible, its kernel contains the torsion part $E^{\text {tor }}$ of $E$, and Manin's theorem of the kernel asserts in this case that

$$
\operatorname{Ker}\left(\boldsymbol{\mu}_{E}\right)=E^{\text {tor }}(S) .
$$

The formula above shows that $\boldsymbol{\mu}_{E}$ extends to any differential extension $(F, \partial)$ of $K$. In the language of differential algebraic groups (see [9]), $\boldsymbol{\mu}_{E}$ : $E \rightarrow \mathbb{G}_{a}$ is a differential algebraic additive character of order 2, defined over $K$. Its kernel over $F$ may strictly contain $E^{\text {tor }}(F)$ : for instance, the transcendental section $y(t)$ defined, locally and in standard notation, by $\wp\left(\omega_{1}(t)+\sqrt{2} \omega_{2}(t)\right)$ is killed by $\boldsymbol{\mu}_{E}$.

\subsection{Link with Painlevé VI}

Apart from Proposition 1.1 below, this section follows [20]. For links with the Galois theory of Painlevé equations, see [21] (and [10] for the PicardPainlevé solution mentioned just above). 
The $\mathrm{P}$ VI equations depend on four complex parameters $\alpha, \beta, \gamma, \delta$, and are given by

$$
\begin{aligned}
\frac{\mathrm{d}^{2} \xi}{\mathrm{d} t^{2}}=\frac{1}{2}\left(\frac{1}{\xi}+\right. & \left.\frac{1}{\xi-1}+\frac{1}{\xi-t}\right)\left(\frac{\mathrm{d} \xi}{\mathrm{d} t}\right)^{2}-\left(\frac{1}{t}+\frac{1}{t-1}+\frac{1}{\xi-t}\right) \frac{\mathrm{d} \xi}{\mathrm{d} t} \\
& +\frac{\xi(\xi-1)(\xi-t)}{t^{2}(t-1)^{2}}\left(\alpha+\beta \frac{t}{\xi^{2}}+\gamma \frac{t-1}{(\xi-1)^{2}}+\delta \frac{t(t-1)}{(\xi-t)^{2}}\right) .
\end{aligned}
$$

Following R. Fuchs and Manin, this is rewriten in terms of points $y=(\xi, \eta)$ in $E(F)$ as:

$$
t(1-t) \boldsymbol{\mu}_{E}(y)=\alpha \eta+\beta t \frac{\eta}{\xi^{2}}+\gamma(t-1) \frac{\eta}{(\xi-1)^{2}}+\left(\delta-\frac{1}{2}\right) t(t-1) \frac{\eta}{(\xi-t)^{2}} .
$$

Set $\kappa=\left(\kappa_{0}, \kappa_{1}, \kappa_{2}, \kappa_{3}\right):=\left(\alpha,-\beta, \gamma, \frac{1}{2}-\delta\right)$, let $e_{0}=0_{E}, e_{1}=(0,0), e_{2}=$ $(1,0), e_{3}=(t, 0)$ be the 2 -torsion of $E(S)$, and consider the rational function $\psi_{\kappa}$ on $E$ given by

$$
y \longmapsto \psi_{\kappa}(y)=\frac{1}{t(t-1)} \sum_{i=0}^{3} \kappa_{i} \eta\left(y+e_{i}\right) .
$$

Then, P VI finally becomes: find a local section $y(t)$ of $E / S^{\text {an }}$ such that

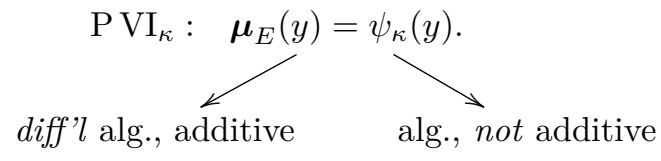

The following result reflects the incompatibility of these properties. The statement itself is inspired by the theme of "unlikely intersections" in diophantine geometry.

Proposition 1.1. - For any finite extension $K^{\prime}$ of $\mathbb{C}(t)$, there are only finitely many points $y=(\xi, \eta) \in E\left(K^{\prime}\right)$ such that $\xi$ solves some (unspecified) PVI equation.

Proof. - We will rely on the following facts from Manin's Mordell paper.

(i) By Mordell-Weil, the set $\left\{\boldsymbol{\mu}_{E}(y), y \in E\left(K^{\prime}\right)\right\}$ lies in a finite dimensional $\mathbb{C}$-subspace of $K^{\prime}$, while the set $\left\{y \in E\left(K^{\prime}\right), \boldsymbol{\mu}_{E}(y)=0\right\}$ is finite; indeed, by the theorem of the kernel, the latter one coincides with $E^{\text {tor }}\left(K^{\prime}\right)$, which is finite.

(ii) (cf. $[18, \S 7$, Prop. 6]). Let $V$ be a finite dimensional $\mathbb{C}$-vector susbspace of $K^{\prime}(E)$. Then, the set $\left\{y \in E\left(K^{\prime}\right), \exists f^{y} \in V \backslash\{0\}, f^{y}(y)=0\right\}$ has bounded height (and is therefore finite, since $E / S$ is not isoconstant). Notice that once $V$ is fixed, the functions $f^{y}$ of the statement are allowed to depend on $y$. 
To prove the proposition, we may restrict to the points $y \in E\left(K^{\prime}\right)$ such that $\boldsymbol{\mu}_{E}(y) \neq 0$, since in view of the second part of (i), the other ones form a finite set. Let then $V$ be the finite-dimensional subspace of $K^{\prime}(E)$ generated over $\mathbb{C}$ by the 4 elements $\eta\left(\cdot+e_{i}\right)$ of $K^{\prime}(E)$ and by the finitely generated subgroup $\Gamma=t(t-1) \boldsymbol{\mu}_{E}\left(E\left(K^{\prime}\right)\right)$ of $K^{\prime}$. By the formula above, the $\xi$-coordinate of a point $y$ in $E\left(K^{\prime}\right)$ satisfies some $\mathrm{P}$ VI equation only if there exist $\mu^{y} \in \Gamma$ and complex numbers $\kappa_{0}^{y}, \ldots, \kappa_{3}^{y}$, depending on $y$ but not all 0 by our restriction, such that $\kappa_{0}^{y} \eta\left(y+e_{0}\right)+\cdots+\kappa_{3}^{y} \eta\left(y+e_{3}\right)-\mu^{y}=0$. Now, consider the rational function $f^{y}(\cdot)=\sum_{i=0, \ldots, 3} \kappa_{i}^{y} \eta\left(\cdot+e_{i}\right)-\mu^{y}$, which lies in $V$. In view of their poles, 1 and the 4 functions $\eta\left(\cdot+e_{i}\right)$ are linearly independent over $K^{\prime}$, so the function $f^{y}$ is not 0 , and we conclude by (ii).

\section{Manin maps}

This section describes various "Manin maps" occuring in the literature. Their kernels will all be compared in the next section, see Theorem 3.1, which is the main statement of this survey. For questions related to their images, see Section 3.3.

\subsection{The Gauss-Manin connections}

From now on, the setting is as follows: the base is a smooth irreducible affine curve $S$ over the complex numbers $\mathbb{C}$, with function field $K=\mathbb{C}(S)$ and coordinate ring $O_{S}=\mathbb{C}[S]$. The results will be insensitive to finite base changes, which we tacitly perform when needed. We fix a non-zero vector field over $S$, and denote by $\partial$ the associated derivation on $K$. We can assume that the ring $\mathcal{D}$ of differential operators on $S$ is $O_{S}[\partial]$. Given a locally free $\mathcal{O}_{S}$-module $V$ with an (integrable) connection $\nabla_{V}$, we write $D_{V}$, or just $D$, for the contraction of $\nabla_{V}$ with $\partial$. This is as an additive endomorphism of $V$ satisfying the Leibniz rule, which naturally extends to an action of $\mathcal{D}$ on $V$ : if $L=\sum_{i} a_{i} \partial^{i}$, then $L(v)=\sum_{i} a_{i}\left(D_{V}\right)^{i}(v)$. We denote by $\mathbb{1}$ the $D$-module $\mathcal{O}_{S}$, with $\nabla=d$.

\subsubsection{Extensions of $D$-modules}

In the category of $D$-modules, extensions $\left(W, \nabla_{W}\right)$

$$
\begin{aligned}
0 \longrightarrow\left(V, \nabla_{V}\right) \longrightarrow & \left(W, \nabla_{W}\right) \longrightarrow\left(\mathcal{O}_{S}, d\right) \longrightarrow 0 \\
& -1304-
\end{aligned}
$$


of $\mathbb{1}$ by $\left(V, \nabla_{V}\right)$ can be concretely described as follows. For $V$ free of rank $n$, fix a basis of $V$ over $O_{S}$, and choose $w \in W$ projecting to $1 \in O_{S}$. Then, $D_{V}=\partial-B$ for some $B \in \mathfrak{g l}_{n}\left(O_{S}\right)$, while the matrix for $\nabla_{W}$ is $\left(\begin{array}{cc}B & c \\ 0 & 0\end{array}\right) \in \mathfrak{g l}_{n+1}\left(O_{S}\right)$ for the column vector $c=D_{W}(w) \in\left(O_{S}\right)^{n}=V(S)$. A fundamental matrix of solutions of $D_{W}$ has the same shape $\left(\begin{array}{cc}\mathcal{B} & u \\ 0 & 1\end{array}\right)$, where $\mathcal{B}$ is a fundamental matrix of solutions of $D_{V}$, and $u$ is a solution of the inhomogenous system $D_{V}(u)=c$. Replacing $w$ by $w+v$ with $v \in V(S)$ replaces $c$ by $c+D_{V}(v)$, and $u$ by $u+v$. It follows that the isomorphism class of the extension $W$ in $\operatorname{Ext}_{D-\bmod }(\mathbb{1}, V)$ is given by the class of $c$ in $V(S) / D_{V}(V(S))$, or more precisely (cf. [13, Prop. 1.1.1]) in the first cohomology group of the complex $V \stackrel{D_{V}}{\longrightarrow} V$ (in degrees 0 and 1), and we have (cf. [4, §1], at least when $\left.K=\mathbb{C}\left(\mathbb{P}_{1}\right)\right)$ :

$$
\operatorname{Ext}_{D-\bmod }(\mathbb{1}, V) \simeq H^{1}\left(V \stackrel{D_{V}}{\longrightarrow} V\right) \simeq V(S) / D_{V}(V(S)) .
$$

Analytically (cf. [13, Prop. 1.6.1]), this $\mathbb{C}$-vector space is given by $H^{1}\left(S, V^{D}\right)$ for the local system of solutions of $D_{V}$; it has finite dimension, depending only on $n$ and $\chi(S)$ when $\nabla_{V}$ is fuchsian (Deligne's formula, cf. [4, Lem. 2]). Anyway, the extension $W$ splits if and only if $c=D_{V}(v)$ for some $v$ in $V(S)$, or equivalently, if and only if $u$ is a $\mathbb{C}$-linear combination of the columns of the matrix $\mathcal{B}$, up to addition of an element of $\left(O_{S}\right)^{n}$.

Considering the connection $\nabla_{V}^{\vee}$ on the dual of $V$, one deduces that $\operatorname{Ext}_{D-\bmod }(V, \mathbb{1}) \simeq H^{1}\left(V^{\vee} \stackrel{D_{V} \vee}{\longrightarrow} V^{\vee}\right)$. In particular, if $L \in \mathcal{D}$ is a non zero differential operator and $V$ is the dual of $\mathcal{D} / \mathcal{D} L$, an extension of $\mathbb{1}$ by $V$ corresponds to an inhomogeneous equation $L(u)=\mu$, for some $\mu \in O_{S}$, and its isomorphism class is given by the image $\bar{\mu}$ of $\mu$ in $O_{S} / L\left(O_{S}\right)$, cf. [4, Lem. 4]. It splits if and only if $u$ is the sum of a solution of $L(\cdot)=0$ and of an element of $O_{S}$. In reflection to the fact that cyclic vectors do not behave well under the constructions of linear algebra, the case $\mu=0$ should be treated in matrix terms to justify that it represents the trivial extension. Obviously, $\mu=0$ implies that $\bar{\mu}=\overline{0}$. It is a remarkable feature that under further (Hodge theoretic) constraints, the representative 0 of $\overline{0}$ will appear naturally in what follows, cf. Section 2.3, and the end of Section 3.3.

\subsubsection{Gauss-Manin}

Let now $A \rightarrow S$ be an abelian scheme of relative dimension $g$, with generic fiber $A_{K}$. The results will be invariant under isogenies and finite base extensions, which we again tacitly perform when needed. In particular, we can assume that the fixed "part", i.e. the $K / \mathbb{C}$ trace, $A_{0} / \mathbb{C}=\operatorname{Tr}_{K / \mathbb{C}}\left(A_{K}\right)$ 
of $A$ is a direct summand. The group

$$
A_{0}(\mathbb{C})^{\mathrm{div}}=\left\{y \in A(S), \exists 0 \neq k \in \mathbb{Z}, k \cdot y \in A_{0}(\mathbb{C})\right\}
$$

contain the torsion sections, and we call its elements the constant sections of $A / S$.

Let further $y \in A(S) \simeq A_{K}(K)$ be a section of $A / S$, and let $\mathbb{A}_{y}=[\mathbb{Z} \stackrel{1 \mapsto y}{\longrightarrow} A]$ be the associated smooth $S$-1-motive, see [15]. This is an extension of [ $\mathbb{Z} \rightarrow 0]$ by $[0 \rightarrow A]$, or said more briefly of $\mathbb{Z}$ by $A$. To keep track of variances, note that its Cartier dual $\mathbb{A}_{y}^{\vee}$ is the extension of the dual abelian variety $A^{\vee}$ by $\mathbb{G}_{m}$ parametrized by $y \in A^{\vee \vee} \simeq A$.

The Betti realizations of these 1-motives can be described as follows: $T_{B}(A)$ is the kernel of the exponential morphism $\exp _{A}: \operatorname{Lie}(A) \rightarrow A$ over $S^{\text {an }}$, while $T_{B}\left(\mathbb{A}_{y}\right)$ is the inverse image under $\exp _{A}$ of $\mathbb{Z} . y$. As such, they are $\mathbb{Z}$-local systems over $S^{\text {an }}$ of ranks $2 g, 2 g+1$. The de Rham realizations, $T_{\mathrm{d} R}(A), T_{\mathrm{d} R}\left(\mathbb{A}_{y}\right)$ (see $[15,10.1 .7]$, and Section 2.3 below) are $\mathcal{O}_{S^{-}}$ modules, which, after extension to $\mathcal{O}_{S^{\text {an }}}$, become isomorphic to $T_{B}(A) \otimes \mathcal{O}_{S^{\text {an }}}$, $T_{B}\left(\mathbb{A}_{y}\right) \otimes \mathcal{O}_{S}$, and so, carry connections id $\otimes d$. As alluded to in Section 1 , one checks that these actually descend to $T_{\mathrm{d} R}(A), T_{\mathrm{d} R}\left(\mathbb{A}_{y}\right)$, which are therefore endowed with canonical connections $\nabla_{A}, \nabla_{\mathbb{A}_{y}}$, called (together with their duals) the Gauss-Manin connections. By construction, their spaces of horizontal sections are given by the Betti realizations, tensored with $\mathbb{C}$.

We are now ready to define the Manin map, or more accurately, the two types of Manin maps which occur in the literature. For lack of a better phrasing, I will call them differential algebraic (they are Manin's original ones [18], as taken up by Buium [9], see also [8]), and cohomological (as extracted by Coleman [13] from Manin's construction). We will begin with the latter ones, which are easier to describe and lead to sharper results. Note that I call them cohomological not because of de Rham cohomologies (these occur in both types), but because their targets are the $H^{1}$ 's of complexes $V \stackrel{D_{V}}{\longrightarrow} V$.

\subsection{The cohomological Manin maps}

The description of the local system $T_{B}\left(\mathbb{A}_{y}\right)$ shows that $T_{\mathrm{d} R}\left(\mathbb{A}_{y}\right)$ lies in $\operatorname{Ext}_{D-\bmod }\left(\mathbb{1}, T_{\mathrm{d} R}(A)\right)$ :

$$
0 \longrightarrow T_{\mathrm{d} R}(A) \longrightarrow T_{\mathrm{d} R}\left(\mathbb{A}_{y}\right) \longrightarrow\left(\mathcal{O}_{S}, d\right)=\mathbb{1} \longrightarrow 0 .
$$

but it will be more convenient to consider the dual connections on $H_{\mathrm{d} R}^{1}(A)=$ $\left(T_{\mathrm{d} R}(A)\right)^{\vee}, H_{\mathrm{d} R}^{1}\left(\mathbb{A}_{y}\right)=\left(T_{\mathrm{d} R}\left(\mathbb{A}_{y}\right)\right)^{\vee}$. These sit in an exact sequence of $D$ modules:

$$
0 \longrightarrow \mathbb{1} \longrightarrow H_{\mathrm{d} R}^{1}\left(\mathbb{A}_{y}\right) \stackrel{\pi}{\longrightarrow} H_{\mathrm{d} R}^{1}(A) \longrightarrow 0 .
$$


So, $H_{\mathrm{d} R}^{1}\left(\mathbb{A}_{y}\right)$ is a $D$-module extension of $H_{\mathrm{d} R}^{1}(A)$ by $\mathbb{1}$. We denote by $\mathcal{M}(y)$ its isomorphism class in $\operatorname{Ext}_{D-\bmod }\left(H_{\mathrm{d} R}^{1}(A), \mathbb{1}\right.$ ), and by $\mathcal{M}$ (or when needs be, $\left.\mathcal{M}_{A}\right)$ :

$$
\begin{aligned}
\mathcal{M}: A(S) \ni y \longmapsto \mathcal{M}(y):=\left[H_{\mathrm{d} R}^{1}\left(\mathbb{A}_{y}\right)\right] \in & \operatorname{Ext}_{D-\bmod }\left(H_{\mathrm{d} R}^{1}(A), \mathbb{1}\right) \\
& \simeq T_{\mathrm{d} R}(A)(S) / D\left(T_{\mathrm{d} R}(A)(S)\right)
\end{aligned}
$$

the corresponding map. This cohomological Manin map is $\mathbb{Z}$-linear and takes values in a $\mathbb{C}$-vector space, so the torsion sections lie in its kernel. In fact, if $y$ is a constant section, the $D$-module $\mathcal{M}_{A_{0}}(y)$ is isomorphic to a direct sum of $\mathbb{1}$ 's, and so, $A_{0}(\mathbb{C})^{d i v}$ is always contained in the kernel of $\mathcal{M}_{A}$. In this situation, the theorem of the kernel (see [13, Thm. 1.4.3], and Theorem 3.1(i) below) is:

$$
\operatorname{Ker}(\mathcal{M})=A_{0}(\mathbb{C})^{\mathrm{div}}
$$

In [13], Coleman pointed out that the following sharper statement would suffice to justify Manin's proof of Mordell, cf. [19]. Let $\Omega=H^{0}\left(A, \Omega^{1}(A / S)\right) \hookrightarrow$ $H_{\mathrm{d} R}^{1}(A)$ be the $\mathcal{O}_{S}$-module of relative differential forms of the 1st kind on $A / S$. It is not a $D$-submodule (unless the Kodaira map is trivial, i.e. when $A=A_{0}$, cf. [3]), and we denote by $[\Omega]$ the $D$-submodule of $H_{\mathrm{d} R}^{1}(A)$ generated by $\Omega$. Let $\mathcal{M}_{[[\Omega]}(y) \in \operatorname{Ext}_{D-\bmod }([\Omega], \mathbb{1})$ be the isomorphism class of the restriction to $[\Omega]$ of the extension $\mathcal{M}(y)$. Then

$$
\mathcal{M}_{\mid[\Omega]}: A(S) \ni y \longmapsto \mathcal{M}_{\mid[\Omega]}(y) \in \operatorname{Ext}_{D-\bmod }([\Omega], \mathbb{1})
$$

is a less precise cohomological Manin map: its kernel clearly contains that of $\mathcal{M}$. Coleman asked and Chai [12] proved that these kernels

$$
\operatorname{Ker}\left(\mathcal{M}_{\mid[\Omega]}\right)=\operatorname{Ker}(\mathcal{M})
$$

actually coincide (see Theorem 3.1(ii)).

More generally, let $i_{V}: V \hookrightarrow H_{\mathrm{d} R}^{1}(A)$ be any $D$-submodule of $H_{\mathrm{d} R}^{1}(A)$. Then $i_{V}^{*}(\mathcal{M}(y))$ gives a map $i_{V}^{*} \circ \mathcal{M}: A(S) \rightarrow \operatorname{Ext}_{D-\bmod }(V, \mathbb{1})$. Chai also considered this broader situation (see [12], but also [5] and [11]), to which we will come back in Section 3.2.

Remark 2.1. - Here is a description of $\mathcal{M}$ in terms of $D$-groups and their logarithmic derivatives, cf. [7]. Recall that a $D$-group is an algebraic group $G / K$ endowed with an extension of $\partial$ to $\mathcal{O}_{G}$ respecting the group structure of $G$. An abelian variety admits a $D$-group structure only if it is constant. On the other hand, the universal vectorial extension $\widetilde{A} \in \operatorname{Ext}_{\text {alg.gr. } / K}\left(A, W_{A}\right)$ of $A$ :

$$
0 \longrightarrow W_{A} \longrightarrow \widetilde{A} \longrightarrow A \longrightarrow 0
$$

with $W_{A}=H^{1}\left(A, \mathcal{O}_{A}\right)^{\vee} \simeq\left(\operatorname{Ext}\left(A, \mathbb{G}_{a}\right)\right)^{\vee}$ of dimension $g$, has a unique $D$ group structure. Now, $T_{\mathrm{d} R} A$ is given by the Lie algebra $L \widetilde{A}$ of $\widetilde{A}$, and this 
allows us to describe the map $\mathcal{M}$ as follows: let $\partial \ell n_{\tilde{A}}: \widetilde{A} \rightarrow L \widetilde{A}$ be the logarithmic derivative on the $D$-group $\widetilde{A}$. This induces on $L \widetilde{A}$ the Gauss-Manin connection $\nabla_{A}$ of $T_{\mathrm{d} R}(A)$, contracted with $\partial$. Now, lift the section $y \in A(S)$ to $\widetilde{y} \in \widetilde{A}(S)$, and consider the projection of $\partial \ell n_{\tilde{A}}(\widetilde{y})$ to $L \widetilde{A}(S) / D_{A}(L \widetilde{A}(S))$. This projection is independent of the choice of the lift (since $\partial \ell n_{\tilde{A}}$ induces $D_{A}$ on the vectorial subgroup $W_{A} \simeq L W_{A}$ ), and by [7], it coincides with $\mathcal{M}(y) \in \operatorname{Ext}_{D-\bmod }\left(H_{\mathrm{d} R}^{1}(A), \mathbb{1}\right)$, which is indeed the cokernel of the connection $\nabla_{A}$ on $H_{\mathrm{d} R}^{1}(A)^{\vee} \simeq T_{\mathrm{d} R}(A) \simeq L \widetilde{A}$.

\subsection{The differential algebraic Manin maps}

Contrary to the previous subsection, the variations of pure, resp. mixed, Hodge structures that $H_{\mathrm{d} R}^{1}(A)$, resp. $H_{\mathrm{d} R}^{1}\left(\mathbb{A}_{y}\right)$, carry will now play a role. Indeed, the exact sequence

$$
0 \longrightarrow \mathbb{1} \longrightarrow H_{\mathrm{d} R}^{1}\left(\mathbb{A}_{y}\right) \stackrel{\pi}{\longrightarrow} H_{\mathrm{d} R}^{1}(A) \longrightarrow 0
$$

is the de Rham realization of an exact sequence of VMHS, and the $\mathcal{O}_{S^{-}}$ module $\Omega:=H^{0}\left(A, \Omega_{A / S}^{1}\right)$ is the Fil $^{1}$ of the Hodge structure (of weight 1) of $H_{\mathrm{d} R}^{1}(A)$. Now, the Fil ${ }^{1}$ of the mixed Hodge structure (of weights 0 and 1 ) carried by $H_{\mathrm{d} R}^{1}\left(\mathbb{A}_{y}\right)$ projects under $\pi$ onto $\mathrm{Fil}^{1}\left(H_{\mathrm{d} R}^{1}(A)\right)$, and they actually have the same rank since the pure Hodge structure of the kernel of $\pi$ has type $(0,0)$. So, there is a canonical section $s_{\Omega}$ of $\pi$ over $\Omega$, and following [13], we will view $\Omega$ as a subspace of both $H_{\mathrm{d} R}^{1}(A)$ and, via $s_{\Omega}$, of $H_{\mathrm{d} R}^{1}\left(\mathbb{A}_{y}\right)$.

Of course, $s_{\Omega}$ will in general not extend to a $\mathcal{D}$-section above the $D_{A^{-}}$ module $[\Omega]$ generated by $\Omega$ in $H_{\mathrm{d} R}^{1}(A)$. When this does occur, we will say that the extension $\mathcal{M}_{\mid[\Omega]}(y)$ of $[\Omega]$ by $\mathbb{1}$ admits a $(D$, Fil)-section. More generally, we may consider the pointed set (actually a group) $\operatorname{Ext}_{D, \text { Fil }}([\Omega], \mathbb{1})$ of isomorphism classes of extensions of $[\Omega]$ by $\mathbb{1}$ endowed with an $\mathcal{O}_{S}$-section above $\Omega$, which admits a forgetful map

$$
\mathcal{F}: \operatorname{Ext}_{D, \mathrm{Fil}}([\Omega], \mathbb{1}) \longrightarrow \operatorname{Ext}_{D-\bmod }([\Omega], \mathbb{1}) .
$$

The map $\mathcal{M}_{\mid[\Omega]}: A(S) \rightarrow \operatorname{Ext}_{D-\bmod }([\Omega], \mathbb{1})$ factors through $\mathcal{F}$ (thanks to the section $s_{\Omega}$ ), and we will see in Theorem 3.1, proof of (iii) (b), that $\mathcal{F}$ is injective on the image of $A(S)$ in $\operatorname{Ext}_{D, \mathrm{Fil}}([\Omega], \mathbb{1})$. In fact, an explicit computation of $\operatorname{Ext}_{D, F i l}([\Omega], \mathbb{1})$ should clarify why the kernel of the Manin map $\boldsymbol{\mu}$ below contains the kernel of $\mathcal{M}_{\mid[\Omega]}$, in the spirit of the discussion on $\mu=0$ and $\bar{\mu}=\overline{0}$ at the end of Section 2.1.1 (see also Section 3.3). But let us come back to the construction of $\boldsymbol{\mu}$.

Via the Gauss-Manin connections, $\mathcal{D}$ acts on $H_{\mathrm{d} R}^{1}(A), H_{\mathrm{d} R}^{1}\left(\mathbb{A}_{y}\right)$, and this defines two maps $\phi, \phi_{y}$ of left $\mathcal{D}$-modules from $\mathcal{D} \otimes_{\mathcal{O}_{S}} \Omega$ to $H_{\mathrm{d} R}^{1}(A), H_{\mathrm{d} R}^{1}\left(\mathbb{A}_{y}\right)$, 
which commute with the projection $\pi$. The image of $\phi$ is [R], and we denote its kernel by $P F$ (for Picard-Fuchs as in [18]). So, $\mathcal{L}=\sum_{i} L_{i} \otimes \omega_{i}$ lies in $P F$ if and only if $\phi(\mathcal{L})=\sum_{i} L_{i}\left(D_{A}\right)\left(\omega_{i}\right)=0 \in H_{\mathrm{d} R}^{1}(A)$. The image of $\phi_{y}$ is $i_{[\Omega]}^{*} \mathcal{M}(y)$. For any $\mathcal{L}$ in $P F, \phi_{y}(\mathcal{L})=\sum_{i} L_{i}\left(D_{\mathbb{A}_{y}}\right)\left(\omega_{i}\right) \in H_{\mathrm{d} R}^{1}\left(\mathbb{A}_{y}\right)$ lies in the kernel $\mathcal{O}_{S}$ of $\pi$, and we define the Manin map $\mu_{\mathcal{L}}: A(S) \rightarrow O_{S}$ attached to $\mathcal{L}$ by:

$$
\mu_{\mathcal{L}}: A(S) \ni y \longmapsto \mu_{\mathcal{L}}(y):=\phi_{y}(\mathcal{L}) \in O_{S},
$$

The following commutative diagram illustrates this construction. The section $y \in A(S)$ is given, and the first vertical arrow is the map $\mathcal{L} \rightarrow \mu_{\mathcal{L}}(y)$ induced by $\phi_{y}$ on $P F$.

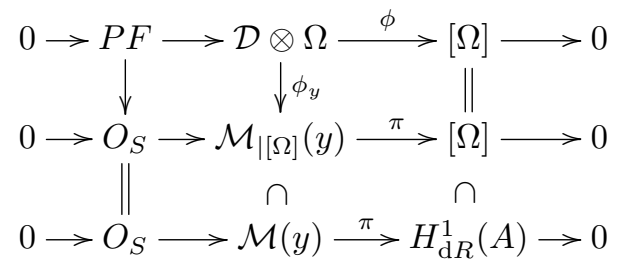

For any $\mathcal{L}$ in $P F$, the map $\mu_{\mathcal{L}}$ is additive in $y$. When $\mathcal{L}$ runs through $P F$, their collection measures how far $\mathcal{M}_{\mid[\Omega]}(y)$ is from being trivial in $\operatorname{Ext}_{D, \text { Fil }}([\Omega], \mathbb{1})$. Since the $\mathcal{D}$-module $P F$ admits a finite number of generators, say $\mathcal{L}_{1}, \ldots, \mathcal{L}_{N}$, we may group all the information on $P F$ by defining the differential algebraic Manin map as the map

$$
\boldsymbol{\mu}_{A}:=\boldsymbol{\mu}=\left(\mu_{\mathcal{L}_{1}}, \ldots, \mu_{\mathcal{L}_{N}}\right): A(S) \longrightarrow\left(\mathbb{G}_{a}\right)^{N}(S) .
$$

Just like $\boldsymbol{\mu}_{E}$ in Section 1, this a differential algebraic additive morphism, and the initial version of Manin's theorem of the kernel (see [18, Thm. 2], and $[13$, Thm. 2.1.0]) states that

$$
\operatorname{Ker}(\boldsymbol{\mu})=A_{0}(\mathbb{C})^{d i v} .
$$

For the intermediate step $\operatorname{Ker}(\boldsymbol{\mu})=\operatorname{Ker}\left(\mathcal{M}_{\mid[\Omega]}\right)$ towards this conclusion, see Theorem 3.1 (iii), which, combined with (ii) and (i), yields the full statement.

To compute the maps $\mu_{\mathcal{L}}$, one needs a concrete vizualization of the extension $H_{\mathrm{d} R}^{1}\left(\mathbb{A}_{y}\right)$. We may interpret it as the exact sequence

$$
0 \longrightarrow \mathcal{O}_{S} \longrightarrow H_{\mathrm{d} R}^{1}\left(A / S, Z_{y}\right) \stackrel{\pi}{\longrightarrow} H_{\mathrm{d} R}^{1}(A / S) \longrightarrow 0,
$$

where the middle term is the relative cohomology with respect to the subscheme $Z_{y}$ of $A / S$ formed by the union of the image of $y$ and of the zero section: if a relative differential $\xi$ (regular along 0 and $y$ ) projects to 0 in $H_{\mathrm{d} R}^{1}(A / S)$, then $\xi=\mathrm{d} f, f \in K(A)$, is represented by $f(y)-f(0) \in \mathcal{O}_{S}$. Roughly speaking, $H_{\mathrm{d} R}^{1}\left(A / S, Z_{y}\right)$ is the space of differentials of the 2 nd kind on $A$, modded out by exact forms in the "narrow" sense ( $\mathrm{d} f$ with $f(0)=f(y)=0)$, while one mods out by all exact forms to get $H_{\mathrm{d} R}^{1}(A / S)$. 
For instance, suppose that $\mathcal{L}=L_{\omega} \otimes \omega$, where $\omega \in \Omega$ and $L_{\omega} \in \mathcal{D}$ is the annihilator of $\omega$ (viewed in the $\mathcal{D}$-module $H_{\mathrm{d} R}^{1}(A / S)$, hence of order $\leqslant 2 g$ ). Then, an analytic expression for $\mu_{\mathcal{L}}(y)$ is given by

$$
\mu_{L_{\omega} \otimes \omega}(y)=L_{\omega}\left(\int_{0}^{y} \omega\right) .
$$

To get an algebraic expression, we write the extension of $\partial$ to $K(A)$ and to the differential forms on $A_{K}$ in terms of an "admissible" set of parameters at 0 and $y$, as explained in $\left[18\right.$, p. 223]. In this case, $\mu_{\mathcal{L}}(y)$ is just $f(y)-f(0)$, where $L_{\omega}\left(D_{\mathbb{A}_{y}}\right)(\omega)=\mathrm{d} f$. In general, $\partial$ does not commute with $\int_{0}^{y}$, and as in Section 1, fudge factors must be added, to take into account differentiation of the boundary, but this suffices to show that as a function of $y, \mu_{\mathcal{L}}$ extends to a differential $K$-rational additive character of order $\leqslant 2 g$.

This brings us to another type of differential algebraic Manin map, namely: instead of looking at a set of generators of $P F$ as was done in the definition $\boldsymbol{\mu}$, we merely consider the "cyclic" elements of the above type $\mathcal{L}_{\omega}=L_{\omega} \otimes \omega, \omega \in \Omega$. Fixing a basis $\omega_{1}, \ldots, \omega_{g}$ of $\Omega$ over $\mathcal{O}_{S}$, we set

$$
\boldsymbol{\mu}_{\Omega}: A(S) \longrightarrow\left(\mathbb{G}_{a}\right)^{g}(S): y \longmapsto\left(\mu_{\mathcal{L}_{\omega_{i}}}(y), i=1, \ldots, g\right) .
$$

Clearly, the kernel of $\boldsymbol{\mu}$ is contained in that of $\boldsymbol{\mu}_{\Omega}$. As a consequence of the semi-simplicity of the $D$-module $H_{\mathrm{d} R}^{1}(A / S)$, we will see in Theorem 3.1 (vi) that in fact

$$
\operatorname{Ker}\left(\boldsymbol{\mu}_{\Omega}\right)=\operatorname{Ker}(\boldsymbol{\mu}),
$$

although $\mathcal{L}_{\omega_{1}}, \ldots, \mathcal{L}_{\omega_{g}}$ need not generate the full $\mathcal{D}$-module $P F$.

Remark 2.2. - In the spirit of Remark 2.1, but now in the language of differential algebraic groups, here is another presentation of the map $\boldsymbol{\mu}_{\Omega}$. Let again $\widetilde{A}$ be the universal vectorial extension of $A$, equipped with its unique $D$-group structure, and let $W_{A}$ be the unipotent part of $\widetilde{A}$. We have the differential algebraic homomorphism $\partial \ell n_{\tilde{A}}: \widetilde{A} \rightarrow L \widetilde{A}$, surjective over a differential closure of $K$. Here again, if $\widetilde{y} \in \widetilde{A}$ lifts $y \in A$, the image of $\widetilde{y}$ under $\partial \ell n_{\tilde{A}}$, now modulo the subgroup $\partial \ell n_{\tilde{A}}\left(W_{A}\right)$, depends only on $y$. This gives a surjective differential algebraic homomorphism $\boldsymbol{\mu}_{\Omega}^{\prime}: A \rightarrow L \widetilde{A} / \partial \ell n_{\tilde{A}}\left(W_{A}\right)$, which is defined over $K$. This map has the same type of target as $\boldsymbol{\mu}$. Indeed, as pointed out by Pillay in $[8, \S 2.4]$, the quotient $L \widetilde{A} / \partial \ell n_{\tilde{A}}\left(W_{A}\right)$ is a unipotent commutative differential algebraic group, and so, by results of Buium and Cassidy, is differential-algebraically isomorphic to some power of $\mathbb{G}_{a}$. Moreover, the power $\left(\mathbb{G}_{a}\right)^{g}$ suffices, so we may view $\boldsymbol{\mu}_{\Omega}^{\prime}$ as a version of our map $\boldsymbol{\mu}_{\Omega}$. It is shown in [7, Cor. K.3], that at the level of $K$-points, $\operatorname{Ker}\left(\boldsymbol{\mu}_{\Omega}^{\prime}\right)=A_{0}(\mathbb{C})^{d i v}$. 


\section{Manin kernels}

\subsection{The theorems of the kernel}

We now turn to the promised Theorem 3.1, which (except maybe for (vi), and for the injectivity of $\mathcal{F}$ in the proof of (iii)) is a combination of the work of Manin, Coleman, Chai and André.

We recall the notation of Section 2, including the notation $s_{\Omega}$ for the canonical section of $\mathcal{M}(y)$ above $\Omega$. In (iv), (v) and tacitly (vi), $\underline{\omega}$, resp. $\underline{\eta}$, denote a basis $\left\{\omega_{1}, \ldots, \omega_{g}\right\}$ of $\Omega$, resp. differentials of the 2 nd kind $\left\{\eta_{1}, \ldots, \eta_{g}\right\}$ representing a complement of $\underline{\omega}$ into a basis of $H_{\mathrm{d} R}^{1}(A / S)$, while $\left\{\gamma_{1}, \ldots, \gamma_{2 g}\right\}$ is a basis of the Betti homology $T_{B}(A)$.

TheOREM 3.1. - Let $y$ be a section of $A / S$. Then,

(i) $\operatorname{Ker}(\mathcal{M})=A_{0}(\mathbb{C})^{\text {div }}$, i.e. $\mathcal{M}(y)$ is split if and only if $y$ is a constant section.

(ii) $\operatorname{Ker}\left(\mathcal{M}_{\mid[\Omega]}\right)=\operatorname{Ker}(\mathcal{M})$, i.e. $i_{[\Omega]}^{*}$ is injective on the image of $\mathcal{M}$;

(iii) $\operatorname{Ker}(\boldsymbol{\mu})=\operatorname{Ker}\left(\mathcal{M}_{\mid[\Omega]}\right)$.

(iv) $\mathcal{M}(y)=0 \Leftrightarrow \int_{0}^{y}(\underline{\omega}, \underline{\eta})$ is a $\mathbb{C}$-linear combination of the vectors $\int_{\gamma_{j}}(\underline{\omega}, \underline{\eta}), j=1, \ldots, 2 g$, up to addition of an element of $\left(O_{S}\right)^{2 g}$.

(v) $\boldsymbol{\mu}(y)=0 \Leftrightarrow \int_{0}^{y}(\underline{\omega})$ is a $\mathbb{C}$-linear combination of the vectors $\int_{\gamma_{j}}(\underline{\omega}), j=$ $1, \ldots, 2 g$.

(vi) $\operatorname{Ker}\left(\boldsymbol{\mu}_{\Omega}\right)=\operatorname{Ker}\left(\mathcal{M}_{\mid[\Omega]}\right)$.

So, at long last, the four "Manin kernels"

$$
\operatorname{Ker}(\boldsymbol{\mu}) \subset \operatorname{Ker}\left(\boldsymbol{\mu}_{\Omega}\right), \quad \operatorname{Ker}(\mathcal{M}) \subset \operatorname{Ker}\left(\mathcal{M}_{\mid[\Omega]}\right)
$$

coincide: they are all equal to $\left(A_{0}(\mathbb{C})\right)^{\text {div }}$. In particular, they are reduced to the torsion subgroup $A^{\text {tor }}(S)$ when the $K / \mathbb{C}$-trace of $A$ vanishes

Notice that the first two kernels are a priori much smaller than the last two ones. In the elliptic situation of Section 1 (where $[\Omega]$ is the full $\left.H_{\mathrm{d} R}^{1}(E / S)\right), \operatorname{Ker}(\mathcal{M})=\operatorname{Ker}\left(\mathcal{M}_{\mid[\Omega]}\right)$ consists of those $y \in E(S)$ such that $\boldsymbol{\mu}_{E}(y)=L(f)$ for some element $f \in O_{S}$, whereas $\operatorname{Ker}\left(\boldsymbol{\mu}_{E}\right)$ (here obviously equal to $\left.\operatorname{Ker}\left(\boldsymbol{\mu}_{\Omega}\right)\right)$ consists of those $y$ such that $\boldsymbol{\mu}_{E}(y)$ is"physically" equal to the zero of $O_{S}$.

Notice also that when $[\Omega]$ does not fill up $H_{\mathrm{d} R}^{1}(A / S)$, the third kernel is a priori truly smaller than the last one. On the other hand, up to the discrepancy just mentioned between "isomorphic to 0" and "physically 0", the first two ones have the same flavour as the last one: they solely concern the restriction to $[\Omega]$ of $\mathcal{M}(y)$. 


\section{A guide to the proofs of Theorem 3.1}

The statement of Theorem 3.1, and the proofs recorded or given below, have logical overlaps, and could be described in a much smoother way. Our aim here is to remain as close as possible to the literature, in particular Manin's original paper [18], Coleman's reformulation [13], Chai's sharpening [12] and André's presentation [2].

Proof of $(i)$. - This formulation of Manin's theorem is due to Coleman [13, Thm. 1.4.3], who gave two proofs, one algebraic, the other analytic. See also [7, Prop. J.2] for a proof in the setting of Remark 2.1.

Proof of (ii). - This is due to Chai, who gives a Hodge theoretical proof at the end of his paper [12]. See also [7, Thm. K.1] for a proof in the language of Remark 2.1. We will discuss in Section 3.2 below Chai's generalization of (ii), where $[\Omega]$ is replaced by any $D$-submodule $V$ of $H_{\mathrm{d} R}^{1}(A / S)$.

\section{Proof of (iii). -}

(a). - Let us first prove that if $\boldsymbol{\mu}(y)=0$, then $s_{\Omega}$ extends to a $(D$, Fil)section of $\mathcal{M}_{\mid[\Omega]}(y)$ (hence to a $D$-section, hence $\left.\mathcal{M}_{\mid[\Omega]}(y)=0\right)$. Recall the maps $\phi, \phi_{y}$ of Section 2.3 , and assume that $\mu_{\mathcal{L}}(y)=0$ for all $\mathcal{L} \in P F$. For $w$ in $[\Omega]$, choose $\mathcal{L} \in \mathcal{D} \otimes \Omega$ such that $w=\phi(\mathcal{L})$. If $\mathcal{L}^{\prime}$ is another choice, then $\mathcal{L}-\mathcal{L}^{\prime}$ lies in $P F$, and its image under $\phi$ vanishes. So, $\widetilde{w}:=\phi_{y}(\mathcal{L}):=\widetilde{s}(w)$ gives a well-defined section $\widetilde{s}$ of $\pi$ over $[\Omega]$, extending $s_{\Omega}$. And since $\phi$ and $\phi_{y}$ are $\mathcal{D}$-linear, so is $\widetilde{s}$.

The converse statement is easy, and the equivalence is essentially Coleman's Proposition 2.1.1 of [13]. But to finish up the proof of (iii), we must show that if the extension $\mathcal{M}_{\mid[\Omega]}(y)$ admits a $\mathcal{D}$-section, not necessarily inducing $s_{\Omega}$ on $\Omega$, then $\boldsymbol{\mu}(y)=0$; and so, in the end, it does admit a $(D$, Fil)section. In other words, the forgetful map $\mathcal{F}$ introduced in Section 2.3 is injective on the image of $A(S)$ in $\operatorname{Ext}_{D, \text { Fil }}([\Omega], \mathbb{1})$.

(b). - Assuming now that $\mathcal{M}_{\mid[\Omega]}(y)=0$, let us show that $\boldsymbol{\mu}(y)=0$. I do not see a direct proof (again because of the discrepancy between physically 0 and isomorphic to 0 ), but we deduce from (ii) and (i) that $y$ is a constant section and are thereby reduced to the case where $A=A_{0}$ is defined over $\mathbb{C}$, with a basis of $\Omega$ also defined over $\mathbb{C}$. Then, by Abel Jacobi (cf. $[3,3.1]$ ), the constant vector $\int_{0}^{y}(\underline{\omega})$ is a linear combination over $\mathbb{C}$ (even, over $\mathbb{R}$ ) of the vectors $\int_{\gamma_{j}}(\underline{\omega}), j=1, \ldots, 2 g$.

So, let us assume that there exist complex numbers $a_{1}, \ldots, a_{2 g}$ such that for all $i=1, \ldots, g, \int_{0}^{y} \omega_{i}=\sum_{j=1, \ldots, 2 g} a_{j} \int_{\gamma_{j}} \omega_{i}$, and let $\mathcal{L}=\sum_{i=1, \ldots, g} L_{i} \otimes \omega_{i}$ be an element of $P F$. Then, $\phi_{y}(\mathcal{L})$ is represented by an exact form $\mathrm{d} f$, and 


$$
\begin{aligned}
\mu_{\mathcal{L}}(y)=\sum_{i} L_{i}\left(\int_{0}^{y} \omega_{i}\right) \text { is equal to } & \\
\mu_{\mathcal{L}}(y)=\sum_{i} \sum_{j} a_{j} L_{i}\left(\int_{\gamma_{j}} \omega_{i}\right) & =\sum_{j} a_{j}\left(\sum_{i} L_{i}\left(\int_{\gamma_{j}} \omega_{i}\right)\right) \\
& =\sum_{j} a_{j} \int_{\gamma_{j}} \sum_{i} L_{i}\left(\omega_{i}\right)=\sum_{j} a_{j} \int_{\gamma_{j}} \mathrm{~d} f=0
\end{aligned}
$$

(for the third equality, recall that $\partial$ commute with $\int_{\gamma_{j}}$ ). So indeed, $\boldsymbol{\mu}(y)=0$.

Proof of (iv). - The collection of these vectors forms a fundamental matrix of solutions for the differential system attached to the Gauss-Manin connection $\nabla_{A}$ on $H_{\mathrm{d} R}^{1}(A / S)$. As said in Section 2.1.1, the condition on the right expresses the fact that the extension $\nabla_{\mathbb{A}_{y}}$ of $\nabla_{A}$ by $\mathbb{1}$ is split. Note that this condition was incorrectly stated in [2, Thm. 1.1.(ii)], where the proviso "up to addition of an element of $\left(O_{S}\right)^{2 g}$ " must be added. On the other hand, its Condition (iii) was correctly stated, and does imply the corrected version of (ii) when $[\Omega]$ fills up $H_{\mathrm{d} R}^{1}(A / S)$, bearing in mind the fudge factors due to the derivations of the boundaries of the integrals.

Proof of $(v)$. - (It is essentially Proposition 4 of Manin's paper [18].) If $\boldsymbol{\mu}(y)=0$, the combination of (iii), (ii) and (i) implies that $y$ is a constant section, and we conclude by Abel-Jacobi. The converse has just been proved in the second part of Point (iii)(b) above.

Proof of (vi). - If $\mathcal{M}_{\mid[\Omega]}(y)=0, \boldsymbol{\mu}(y)=0$ by (iii), and in particular, $\boldsymbol{\mu}_{\Omega}(y)=0$. Conversely, assume that $\boldsymbol{\mu}_{\Omega}(y)=0$. For any $\omega_{i}$ in a basis of $\Omega$, with annihilator $L_{\omega_{i}} \in \mathcal{D}$, this implies that the restriction $\mathcal{M}_{\mid\left[\omega_{i}\right]}(y)$ of $\mathcal{M}_{\mid[\Omega]}(y)$ to the $D$-submodule $\left[\omega_{i}\right] \simeq \mathcal{D} / \mathcal{D} L_{\omega_{i}}$ generated by $\omega_{i}$ in $H_{\mathrm{d} R}^{1}(A / S)$ is split. Now, a fundamental result of Deligne [14, Thm. 4.2.6], states that the $D$-module $H_{\mathrm{d} R}^{1}(A / S)$ is semi-simple. Consequently, one can speak of the largest D-submodule $U$ of $H_{\mathrm{d} R}^{1}(A / S)$ over which the extension $\mathcal{M}(y)$ is split (see for instance $[17, \S 1.2 .1]$, for the dual statement). Since the $\left[\omega_{i}\right]$ 's generate $[\Omega], U$ contains $[\Omega]$, and the restriction $\mathcal{M}_{\mid[\Omega]}(y)$ of $\mathcal{M}(y)$ to $[\Omega]$ is split.

\subsection{Chai's theorem}

In order to prove Part (ii) of Theorem 3.1, namely that $\mathcal{M}(y)$ splits if (and clearly only if) its restriction to $[\Omega]$ does, one can assume that the abelian scheme $A / S$ is geometrically simple. Indeed, for $A$ isogenous to $A_{1} \times A_{2}$, one has in obvious notation: $\mathcal{M}_{\mid[\Omega]} \simeq \mathcal{M}_{\mid\left[\Omega_{1}\right]} \times \mathcal{M}_{\mid\left[\Omega_{2}\right]}$, and similarly with 
$\mathcal{M}_{A_{1} \times A_{2}}$. So in this subsection, we will assume that $A / S$ is geometrically simple. In particular, either $A / S$ is constant, or its $K / \mathbb{C}$-trace $A_{0}$ vanishes. Chai then proposed in [12] the following generalization of his Theorem 3.1(ii).

TheOREm 3.2. - Let $A / S$ be a simple abelian scheme, let $y \in A(S)$ be a section, and let $i_{V}: V \hookrightarrow H_{\mathrm{d} R}^{1}(A)$ be a non-zero $D$-submodule of $H_{\mathrm{d} R}^{1}(A)$. Assume that $i_{V}^{*}(\mathcal{M}(y)):=\mathcal{M}_{\mid V}(y) \in \operatorname{Ext}_{D-\bmod }(V, \mathbb{1})$ is split. Then, $\mathcal{M}(y)$ is split.

Unfortunately, the proof given in [12] relies on the assumption that if the simple abelian scheme $A / S$ is not constant, then the $D$-module $H_{\mathrm{d} R}^{1}(A)$ is irreducible. This is not always the case, as shown by Deligne's counterexample in [16]. In [5] (see also [11]), I pointed out this gap, and proposed a way to fix it. This corrected proof, reproduced in its original form, is the content of what follows. For other presentations, see [6, Formulae $\left(2^{\prime}\right),\left(2^{*}\right)$ (in the setting of Remark 1)], and André's report [2], which incorporates a proof of his results [1] on algebraic independence.

It will here be more convenient to return to the covariant view-point of Section 2.1.2. So, given a $D$-submodule $V$ of $T_{\mathrm{d} R}(A)$ strictly contained in $T_{\mathrm{d} R}(A)$, i.e. with a non-trivial projection $p:=p_{V}: T_{\mathrm{d} R}(A) \rightarrow T_{\mathrm{d} R}(A) / V$, we must show that if the $D$-module

$$
p_{*}\left(T_{\mathrm{d} R}\left(\mathbb{A}_{y}\right)\right)=T_{\mathrm{d} R}\left(\mathbb{A}_{y}\right) / V \in \operatorname{Ext}_{D-\bmod }\left(\mathbb{1}, T_{\mathrm{d} R}(A) / V\right)
$$

is a split extension, then $T_{\mathrm{d} R}\left(\mathbb{A}_{y}\right) \in \operatorname{Ext}_{D-\bmod }\left(\mathbb{1}, T_{\mathrm{d} R}(A)\right)$ is split.

Proof. - Fix a point $s \in S$, and let $T_{A, s}, T_{A, y, s}$ be the fiber at $s$ of the local systems $T_{B}(A), T_{B}\left(\mathbb{A}_{y}\right)$ (tensored with $\mathbb{Q}$ ). These fibers define an extension

$$
0 \longrightarrow T_{A, s} \longrightarrow T_{A, y, s} \longrightarrow \mathbb{Q} \longrightarrow 0
$$

of representations over $\mathbb{Q}$ of the fundamental group $\pi_{1}(S, s)$. Here, $\mathbb{Q}$ is the trivial representation. Since all our differential equations are fuchsian at the missing points of $S$, this extension of monodromy representations splits if and only if $T_{\mathrm{d} R}\left(\mathbb{A}_{y}\right)$ splits.

Chai's argument [12] now goes as follows. We consider the following algebraic groups over $\mathbb{Q}$ :

- $\widetilde{G} \subset G L\left(T_{A, y, s}\right)$ is the $\mathbb{Q}$-Zariski closure of the image of $\pi_{1}$ acting on $T_{A, y, s}$; this group depends on $y$;

- $G \subset G L\left(T_{A, s}\right)$ is the $\mathbb{Q}$-Zariski closure of the image of $\pi_{1}$ acting on $T_{A, s}$; by [14], the (connected component $G^{0}$ of the) group $G$ is a reductive group;

- $N=$ kernel of the natural map $\widetilde{G} \rightarrow G$; the construction below shows that $N$ is abelian, hence acted upon naturally by $\widetilde{G} / N=G$. 
Fixing apoint $\tilde{\lambda} \in T_{A, y, s}$ above $1 \in \mathbb{Q}$, and considering $g \widetilde{\lambda}-\widetilde{\lambda}$, we obtain a cocycle $\xi_{y} \in H^{1}\left(\widetilde{G}, T_{A, s}\right)$, whose restriction to $N$

$$
\xi(y): N \longrightarrow T_{A, s}
$$

is a $G$-equivariant injective morphism between vectorial groups over $\mathbb{Q}$. So, $N$ identifies with a $\mathbb{Q}[G]$-submodule of $T_{A, s}$. Since $G$ is reductive, $N=0$ if and only if the extension $T_{A, y, s}$ (equivalently $T_{\mathrm{d} R}\left(\mathbb{A}_{y}\right)$ ) splits: indeed, $T_{A, y, s}$ becomes a representation of $G$ if $N=0$.

By hypothesis, $V$ is a strict $D$-submodule of $T_{\mathrm{d} R}(A)$ such that $T_{\mathrm{d} R}\left(\mathbb{A}_{y}\right) / V$ splits as a $D$-module extension of $\mathbb{1}$ by $T_{\mathrm{d} R}(A) / V$. Since the $\mathbb{C}$-local system $T(V)$ of horizontal vectors of $V$ needs not be generated by its intersection with the $\mathbb{Q}$-structure $T_{B}(A)$, we extend the scalars to $\mathbb{C}$, and consider the projection of $N$ to $\left(T_{A, s} \otimes \mathbb{C}\right) /\left(T(V)_{s}\right.$. Since $T_{\mathrm{d} R}\left(\mathbb{A}_{y}\right) / V$ splits, one easily checks that this projection vanishes. So, $N \otimes \mathbb{C} \subset(T(V))_{s}$ does not fill up $T_{A, s} \otimes \mathbb{C}$, and $N$ must be a strict $\mathbb{Q}[G]$-submodule of $T_{A, s}$.

If $T_{A, s}$ is an irreducible $\mathbb{Q}[G]$-module (equivalently, if $T_{\mathrm{d} R}(A)$ is an irreducible $D$-module), this implies that $N=0$, hence $T_{\mathrm{d} R}\left(\mathbb{A}_{y}\right)$ splits, as was to be shown. But as said above, this hypothesis does not always hold, even if the simple abelian scheme $A$ is not constant. To fill up this gap, we will now appeal to André's normality theorem on the Mumford-Tate groups attached to $S$-1-motives (see [1], and the Remark at the end of $[2, \S 1.5]$ for a motivic presentation).

For any $s \in S$, we denote by $M T_{A, s} \subset G L\left(T_{A, s}\right)$ (resp. $M T_{A, y, s} \subset$ $\left.G L\left(T_{A, y, s}\right)\right)$ the Mumford-Tate group of the Hodge structure (resp. mixed HS) attached to $A$ (resp. $\mathbb{A}_{y}$ ). These are connected algebraic groups over $\mathbb{Q}$, which satisfy the following facts:

(1) $\left(\left[1\right.\right.$, Lem. 4]): there is a meager subset of $S$ whose complement $S_{0}$ is pathwise connected, and such that $M T_{A, y, s}$ (hence $M T_{A, s}$ ) is locally constant over $S_{0}$.

(2) ([1, Thm. 1]) Let $\widetilde{G}_{s}^{0}$ be the connected component of the (monodromy) group called $\widetilde{G}$ above. Then, for any $s \in S_{0}, \widetilde{G}_{s}^{0}$ is a normal subgroup of $M T_{A, y, s}$. Actually, [1] further shows that $\widetilde{G}_{s}^{0}$ is contained in the derived group of $M T_{A, y, s}$, but we will not need this sharpening.

After extension to a finite cover of $S$, we may assume that $G$, hence $\widetilde{G}$ are already connected. We make this assumption from now on, and proceed to finish up the proof of Theorem 3.2 (in its dual version). We fix a base point $s$ in $S_{0}$ as in Fact (1), yielding the algebraic groups

- $\widetilde{G}$ as above, normal in $\widetilde{M T}:=M T_{A, y, s}$ by Fact (2); 
- $G$ as above, normal in $M T:=M T_{A, s}$;

- $N$ as above, contained in the kernel

$$
N T=\left\{g \in \widetilde{M T}, g\left(T_{A, y, s}\right) \subset W_{-1}\left(T_{A, y, s}\right)=T_{A, s}\right\}
$$

of the natural map $\widetilde{M T} \rightarrow M T$. Fixing apoint $\widetilde{\lambda} \in T_{A, y, s}$ above $1 \in$ $\mathbb{Q}$, and considering $g \widetilde{\lambda}-\widetilde{\lambda}$, we obtain a cocycle $\Xi_{y} \in H^{1}\left(\widetilde{M T}, T_{A, s}\right)$, whose restriction $\Xi(y): N T \rightarrow T_{A, s}$ to $N T$ shows that $N T$ is abelian (and is a $\mathbb{Q}[M T]$-submodule of $T_{A, s}$ ). Notice for later use that the restriction of $\Xi_{y}, \Xi(y)$, to $\widetilde{G}, N$, coincide with the maps $\xi_{y}, \xi(y)$, of the previous paragraph.

As recorded, $\widetilde{G}$ is normal in $\widetilde{M T}$. We will now show that $N$ too is normal in $\widehat{M T}$. Extending the scalars to $\mathbb{C}$, it suffices to show that $N_{\mathbb{C}}$ is normal in $\widetilde{M T}_{\mathbb{C}}$. Since $G_{\mathbb{C}}$ is reductive and $N_{\mathbb{C}}$ is abelian, $N_{\mathbb{C}}$ is the unipotent radical of $\widetilde{G}_{\mathbb{C}}$, i.e. the (unique) maximal connected unipotent normal subgroup of $\widetilde{G}_{\mathbb{C}}$. Therefore, $N_{\mathbb{C}}$ is fixed under any automorphism of $G_{\mathbb{C}}$, and in particular, under all outer automorphisms $\operatorname{Int}(g), g \in \widetilde{M} T(\mathbb{C})$ of $\widetilde{G}_{\mathbb{C}}$ that the normality of $\widetilde{G}$ in $\widetilde{M T}$ provides. So, $N_{\mathbb{C}}$ is indeed normal in $\widetilde{M T}{ }_{\mathbb{C}}$. And since the abelian group $N T$ acts trivially on its subgroup $N$, the action of $\widetilde{M T}$ on $N$ by conjugation induces an action of $\widetilde{M T} / N T=M T$.

We now see that the $\mathbb{Q}$-morphism

$$
\xi(y)=\left(\Xi_{y}\right)_{\mid N}: N \longrightarrow T_{A, s}
$$

is equivariant not only under $G$, but also under the full action of $M T$. So, $N$ identifies with a $M T$-submodule of $T_{A, s}$. Now, $T_{A, s}$ is irreducible as a $\mathbb{Q}[M T]$-module, since our choice of $s$ forces $\operatorname{End}_{M T}\left(T_{A, s}\right)=\operatorname{End}\left(A_{s}\right)=$ $\operatorname{End}(A / S)$, and we conclude that either $N=0$ (implying as before that $T_{\mathrm{d} R}\left(\mathbb{A}_{y}\right)$ is split), or that $N=T_{A, s}$. As we already saw, the latter case prevents the existence of any non-trivial projection $p$ such that $p_{*}\left(T_{\mathrm{d} R}\left(\mathbb{A}_{y}\right)\right)$ splits, unless $T_{\mathrm{d} R}\left(\mathbb{A}_{y}\right)$ itself is split.

\subsection{Manin images}

Now that the kernels of the Manin maps have been described, we will say a few words on their images. Since their targets are $\mathbb{C}$-vector spaces, it is natural to study their $\mathbb{C}$-linear extensions. The following proposition, borrowed from [8], shows how differently the differential algebraic and cohomological Manin maps behave in this respect. 


\section{Proposition 3.3. -}

(i) There exists a simple traceless abelian scheme $A / S$ such that the $\mathbb{C}$ linear extension $\boldsymbol{\mu}_{\Omega}^{\prime} \otimes 1: A(S) \otimes \mathbb{C} \rightarrow\left(O_{S}\right)^{g}$ of $\boldsymbol{\mu}_{\Omega}^{\prime}$ is not injective.

(ii) If $A / S$ is a traceless abelian scheme, the $\mathbb{C}$-linear extension $\mathcal{M} \otimes 1$ : $A(S) \otimes \mathbb{C} \rightarrow T_{\mathrm{d} R}(A)(S) / D_{A}\left(T_{\mathrm{d} R}(A)(S)\right)$ of $\mathcal{M}$ is injective.

Proof. - (See [8, Prop. 2.14 and 2.15, and their proofs in §5].) The counterexample for (i) is an abelian scheme of relative dimension 4 , whose $H_{\mathrm{d} R}^{1}(A / S)$ strictly contains its $D_{A^{-}}$-submodule $[\Omega]$. The proofs use the settings of Remarks 1 and 2.

Let me close with a tentative approach to the image of the maps $\boldsymbol{\mu}_{\Omega}, \boldsymbol{\mu}_{\Omega}^{\prime}$ : it is tempting to interpret their target $\left(O_{S}\right)^{g}$ as the Lie algebra $\Omega^{\vee}$ of $A / S$. However, the map $\Omega \rightarrow O_{S}$ deduced from our association $\omega \mapsto \mathcal{L}_{\omega}=L_{\omega} \otimes \omega$, composed with $\phi_{y}$ :

$$
\{\omega \in \Omega\} \longrightarrow\left\{\mathcal{L}_{\omega} \in P F\right\} \stackrel{\phi_{y}}{\longrightarrow}\left\{\mu_{\mathcal{L}_{\omega}}(y) \in O_{S}\right\}
$$

is seldom additive in $\omega$ ([13, Prop. 2.1.3] does provide an $O_{S}$-linear map $\Omega \rightarrow$ $P F \stackrel{\phi_{y}}{\longrightarrow} O_{S}$, but only under the condition that the Kodaira map has maximal rank, which implies that $\left.[\Omega]=H_{\mathrm{d} R}^{1}(A / S)\right)$. In any case, $\operatorname{Ext}_{D, \text { Fil }}([\Omega], \mathbb{1})$ should be computable in terms of $[\Omega]^{\vee}$ (or rather, its quotient $\Omega^{\vee}$ ), just like $\operatorname{Ext}_{D-\bmod }([\Omega], \mathbb{1})$ is the quotient of $[\Omega]^{\vee}(S)$ by $D_{A}\left([\Omega]^{\vee}(S)\right)$. This would give a concrete description of the forgetful map $\mathcal{F}$, and a direct link between a differential algebraic map of the type $\boldsymbol{\mu}_{\Omega}$, now with values in $\operatorname{Ext}_{D, \text { Fil }}([\Omega], \mathbb{1})$, and the cohomological map $\mathcal{M}_{\mid[\Omega]}$.

\section{Acknowledgements}

I thank Y. André, C-L. Chai, N. Katz, C. Simpson and C. Voisin for their comments on my note [5], and A. Pillay for the numerous exchanges we had on the definitions of Manin maps. André's report [2] was of great help for the present version, as well as his remarks on Coleman's paper. Finally, I thank Hiroshi Umemura for the inspiration he brought me, sharing not only his expertise, but also his broad views on our mutual cultures.

\section{Bibliography}

[1] Y. ANDRÉ, "Mumford-Tate groups of mixed Hodge structures and the theorem of the fixed part", Compos. Math. 82 (1992), no. 1, p. 1-24. 
[2] - "Groupes de Galois motiviques et périodes", in Séminaire Bourbaki. Volume 2015/2016, Astérisque, vol. 390, Société Mathématique de France, 2015, exposé n 1104, p. 1-26.

[3] Y. André, P. Corvaja \& U. Zannier, "The Betti map associated to a section of an abelian scheme (with an appendix by Z. Gao)", https://arxiv.org/abs/1802.03204, 2018.

[4] D. Bertrand, "Extensions de $D$-modules et groupes de Galois différentiels", in p-adic analysis, Lecture Notes in Mathematics, vol. 1454, Springer, 1990, p. 125-141.

[5] — "Manin's theorem of the kernel : a remark on a paper of C-L. Chai", unpublished, webusers.imj-prg.fr/ daniel.bertrand/, 2008.

[6] - "Galois descent in Galois theories", in Arithmetic and Galois theories of differential equations, Séminaires et Congrès, vol. 23, Société Mathématique de France, 2011, p. 1-24.

[7] D. Bertrand \& A. Pillay, "A Lindemann-Weierstrass Theorem for semiabelian varieties over function fields", J. Am. Math. Soc. 23 (2010), no. 2, p. 491-533.

[8] - "Galois theory, functional Lindemann-Weierstrass and Manin maps", Pac. J. Math. 281 (2016), no. 1, p. 51-82.

[9] A. Buium, Differential algebra and diophantine geometry, Actualités Mathématiques, Hermann, 1994.

[10] G. Casale, "The Galois groupoid of Picard-Painlevé VI equation", RIMS Kôkyûroku Bessatsu B2 (2007), p. 15-20.

[11] C.-L. CHAI, "Correction to [12]", available on www.math.upenn.edu/ chai/papers. html.

[12] , "A note on Manin's theorem of the kernel", Am. J. Math. 113 (1991), no. 3, p. $387-389$.

[13] R. F. Coleman, "Manin's proof of the Mordell conjecture over function fields", Enseign. Math. 36 (1990), no. 3-4, p. 393-427.

[14] P. Deligne, "Théorie de Hodge II", Publ. Math., Inst. Hautes Étud. Sci. 40 (1971), p. $5-58$.

[15] - "Théorie de Hodge III", Publ. Math., Inst. Hautes Étud. Sci. 44 (1974), p. 5-77.

[16] G. Faltings, "Arakelov theorem for abelian varieties", Invent. Math. 73 (1983), p. 337-347.

[17] C. Hardouin, "Unipotent radicals of Tannakian Galois groups in positive characteristic", in Arithmetic and Galois theories of differential equations, Séminaires et Congrès, vol. 23, Société Mathématique de France, 2011, p. 283-299.

[18] Y. I. Manin, "Rational points of algebraic curves over functional fields", Izv. Akad. Nauk SSSR, Ser. Mat. 27 (1963), no. 6, p. 1395-1440, translation in Am. Math. Soc., Transl. 50 (1966), p. 189-234.

[19] - "Letter to the editor", Izv. Akad. Nauk SSSR, Ser. Mat. 53 (1989), no. 2, p. 447-448, translation in Math. USSR, Izv. 34 (1990), n 2, p. 465-466.

[20] - "Sixth Painlevé equation, universal elliptic curve, and mirror of $\mathbb{P}_{2}$ ", Am. Math. Soc., Transl. 186 (1998), no. 39, p. 131-151.

[21] H. Umemura, "Galois theory and Painlevé equations", in Théories asymptotiques et équations de Painlevé, Séminaires et Congrès, vol. 14, Société Mathématique de France, 2006, p. 299-339. 